\title{
Il concetto di vis tra diritto privato e repressione criminale
}

Recibido: 22/02/2021. Aceptado: 22/04/2021.

\section{Riassunto}

Si pongono in rilievo le diverse accezioni del termine vis, il suo significato nell'ambito del diritto privato romano e in quello pubblico, levoluzione del concetto stesso di violenza a partire dall'età repubblicana passando per l'epoca del principato fino alle considerazioni di Isidoro di Siviglia.

Parole chiave: vis, violenza, diritto privato, diritto criminale.

\section{El concepto de vis entre el derecho privado y la represión criminal}

\section{Resumen}

En este trabajo se ponen de relieve los diferentes significados del término vis, su significación en el contexto del derecho privado y público romano y la evolución del concepto mismo de violencia desde la época republicana hasta las consideraciones de Isidoro de Sevilla, pasando por el Principado.

Palabras clave: vis, violencia, derecho privado, derecho penal.

\section{The Concept of Vis between Private Law and Criminal Repression}

\begin{abstract}
In this paper, I will highlight the various connotations of the term vis, specifically its meaning in Roman private law and in public law. In doing so, I will address the evolution of the concept of violence starting in the times of the Republic and throughout the Principate, up to the considerations given by Isidore of Seville.
\end{abstract}


L'occasione di ripensare al concetto di vis/violenza nel mondo romano è nata da due Seminari di studio tenuti nel 2015 tra Napoli e Catania. In quelle giornate, era stata scelta per uno dei manifesti una delle tante rappresentazioni dellepisodio biblico dei "giovani nella fornace", immagine sovente utilizzata nell'iconografia cristiana dei primi tempi, che non aveva ancora accettato raffigurazioni di Gesù, secondo i principi della religione ebraica; si adattavano allora scene mitologiche (come il moscoforo assimilato al buon pastore) e rappresentazioni di esempi di salvezza. ${ }^{2}$ Lepisodio, molto noto, è contenuto nel III libro di Daniele; i tre giovani funzionari ebrei che non si erano voluti prostrare dinanzi a una nuova e gigantesca statua d'oro costruita da Nabucodonosor sono gettati in una fornace ardente ma ne escono vivi mentre gli aguzzini che si trovavano lì vicino vengono bruciati.

La rappresentazione iconografica del supplizio non è un motivo decorativo frequente nell'arte precristiana, ma non stupisce la rappresentazione della violenza del martirio in quanto qui serve a evidenziare lo spirito salvifico del credo cristiano.

I supplizi a Roma, come in tutte le civiltà antiche e in larga misura anche in quelle moderne, erano per lo più pubblici, e dunque la violenza appare, nell'antichità, strettamente connessa alla vita quotidiana. ${ }^{4}$ Eva Cantarella ha mostrato che la possibilità di assistere alle pene corporali e all'esecuzione capitale nell'antichità aveva tra l'altro lo scopo di estinguere la sete di vendetta dei familiari oltre che di costituire un deterrente per gli emulatori.'

Può essere rilevante ricordare che oltre alla decapitazione, durante il principato furono irrogate contro i cittadini pene che un tempo erano riservate ai soli schiavi. La damnatio in crucem, ad bestias, la vivi crematio, definite dalle fonti summa supplicia, furono spettacolarizzate e inflitte in particolare agli humiliores, cittadini di bassa estrazione e schiavi.

Naturalmente è indispensabile accostarsi alle rappresentazioni della violenza nel mondo antico con spirito scevro da sovrastrutture che derivano dai nostri tempi, e tanto più è necessario questo distacco nel momento in cui ci si appresta ad analizzare il concetto di vis nel mondo romano.

Bisogna, infatti, focalizzare l'attenzione sul fatto che il termine vis non ha in sé connotazione prettamente negativa; e rapportarsi al mondo greco non è in questo caso proficuo, perché già alle origini della letteratura ellenica in alcuni versi delle Opere e igiorni Esiodo avvicina in netta antitesi Dike e Hybris (v. 213 ss.):

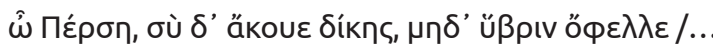

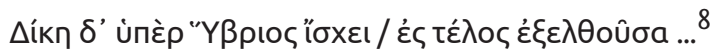

\footnotetext{
1 La violenza nel mondo romano: discipline a confronto (Università di Napoli Federico II, 20 febbraio) e I linguaggi della violenza. Immaginari, pratiche e rappresentazioni tra mondo antico e contemporaneità (Università di Catania, 26-27 novembre). Presento le traduzioni dei soli testi giuridici, che offrono maggiori difficoltà interpretative.

2 Per esempio nell'aretologia martiriale delle catacombe del II secolo d. C. sono assenti immagini dei supplizi cui pure i cristiani erano sottoposti, cfr. McClintock (2015).

3 Cfr. Amodio (2017)

${ }_{4}$ Cfr. a titolo d'esempio, tra i tanti, Vismara (1991).

5 Cantarella (2011, passim e pp. 9-12).

6 Così D. 48.19 .28 pr. (Call. 6 de cogn.); cfr. Pauli Sent. 5.17.2 (3). Cfr. McClintock (2010) pp. 39-40.

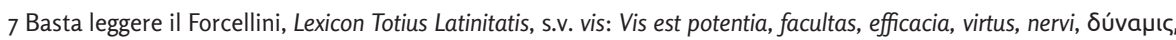
íđxúc (It. Forza, vigore, lena, possa, virtù; Fr. Force, vigueur; Hisp. Fuerza, vigor; Germ. Die Kraft, Stärke, Gewalt; Angl. Force, power, vigour. A) II: Speciatim est violentia... violenza, prepotenza, Bía.

8 Dopo il noto apologo dello Sparviero e l'usignolo, vv. 202 ss., segue la contrapposizione tra giustizia e violenza, vv. 213-237: "O Perse, ascolta la giustizia e non alimentare la prepotenza; ... la giustizia al termine del suo corso vince
} 
Per meglio seguire i percorsi di significato del vocabolo vis può interessare soffermarsi sul dibattito scientifico, rinverdito anche di recente, in merito all'esatta lezione da preferire nelle Istituzioni di Giustiniano a proposito della definizione di tutela;' ius ac potestas in capite libero, infatti, è una definitio che il manuale imperiale fa risalire al giurista tardorepubblicano Servio Sulpicio (1.13.1: Est autem tutela, ut Servius definivit, ius ac potestas in capite libero ad tuendum eum qui propter aetatem se defendere nequit, iure civili data ac permissa).

Nei Digesta giustinianei, invece, nel passo "gemello", l'espressione che si incontra e che risulta tratta da un'opera di Giulio Paolo, è vis ac potestas, ed è questa iunctura che il giureconsulto fa risalire a Servio (D. 26.1.1 pr., Paulus 38 ad edictum): Tutela est, ut Servius definit, vis ac potestas in capite libero ad tuendum eum, qui propter aetatem sua sponte se defendere nequit, iure civili data ac permissa.

Il fatto che nella parafrasi greca delle Istituzioni realizzata da Teofilo poco dopo la com-

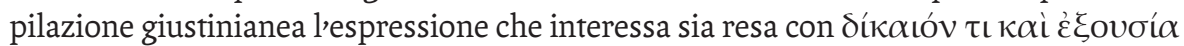

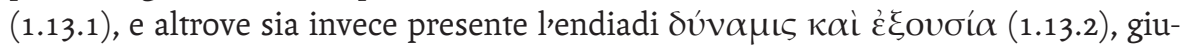
stifica il dubbio anche a prescindere dalle mutate concezioni relative allistituto della tutela prodottesi tra il I secolo a. C., quello in cui visse e operò Servio Sulpicio, e il VI d. C.; la trasformazione, cioè, da una originaria funzione potestativa della figura del tutore, che ricopriva lo stesso ruolo del paterfamilias dal punto di vista dei poteri esercitabili, a quella di "difesa" del pupillo, di attenzione per i suoi interessi e bisogni, corrispondente anche alla diversa concezione della patria potestas. A questo proposito va posto in evidenza che la discussione oggi ripresa da F. Briguglio ${ }^{11}$ sulla lezione corretta del manuale giustinianeo mostra ancora una volta la valenza anfibologica del vocabolo vis in iunctura con potestas.

Con questo si vuole dire che il termine vis ha nel suo interno il significato di violenza ma anche di forza, dal momento che, come abbiamo visto, è assimilabile al vocabolo ius, diritto, e che fin dalla sua comparsa nella lingua latina -è bene precisarlo- non ebbe connotazioni negative, o comunque restò a lungo un termine neutro.

Luigi Labruna (1971) ha dedicato alle origini e alla funzione dellinterdetto uti possidetis - un provvedimento d'urgenza creato dal pretore per la tutela della possessio di fondi, poi di altre res immobili - un importante studio dal titolo Vim fieri veto, che recava come significativo sottotitolo: "Alle radici di una ideologia"; in esso ha illustrato il momento storico nel quale la vis, acquisendo una preponderante valenza negativa, venne concepita come elemento antigiuridico da combattere.

Riprendendo le conclusioni a cui è giunto lo studioso napoletano, che a mio avviso restano ancora ampiamente condivisibili, si osserva che la vis nel suo significato di forza a cui opporsi e da reprimere (e quindi di violenza) da parte del potere statale si manifesta solo a partire, probabilmente, dagli inizi del II secolo a. C., dopo la

la prepotenza..." (trad.it. Magugliani, 1979). Interessanti notazioni sui concetti e i termini in esame in Mittica (2011, pp. 156-169).

9 Sull'istituto, cfr. Viarengo (2015).

10 "La tutela è poi, come la definì Servio, l'autorità e il potere su una persona libera dato e permesso dal diritto civile per proteggere uno che a causa dell'età non può difendersi da solo". Quando non diversamente indicato, le traduzioni sono mie.

11 Briguglio (2010, pp. 163-179); sul concetto di violenza per un quadro generale Balzarini (1993, pp. 830-843); Querzoli (2004, pp. 277-284), con particolare riguardo per il principio di legittima difesa.

12 Cfr. Cic. nat. deor. 2.6o-61: Quicquid enim magnam utilitatem generi adferret humano, id non sine divina bonitate erga homines fieri arbitrabantur. Itaque tum illud quod erat a deo natum nomine ipsius dei nuncupabant, ut cum fruges Cererem appellamus vinum autem Liberum, (...), tum autem res ipsa, in qua vis inest maior aliqua, sic appellatur ut ea ipsa vis nominetur deus, ut Fides ut Mens, quas in Capitolio dedicatas videmus proxume a M. Aemilio Scauro, ante autem ab A. Atilio Calatino erat Fides consecrata. 
fine della seconda guerra punica, quando fu creato appunto linterdetto uti possidetis, che peraltro si limitava a tutelare il possessore da atti violenti compiuti da altri per impedirgli di godere del possesso di un fondo (vim fieri veto); solo più tardi si limitò la tutela a chi possedeva un fondo che fosse stato acquisito senza violenza (Uti nunc possidetis eum fundum quo de agitur, quominus ita possideatis, adversus ea vim fieri veto; "come possedete ora quel fondo di cui si tratta, perché così lo possediate, vieto si faccia violenza contro questa situazione possessoria" poi si aggiunse: eum fundum [...] quod nec vi, nec clam nec precario alter ab altero possidetis, ita possideatis, adversus etc. "quel fondo [...] che non possedete l'uno dall'altro né con la violenza, né di nascosto né a titolo di precario..."). ${ }^{13}$ Le più copiose attestazioni di questa valenza oramai assunta dal vocabolo si possono leggere in alcune orazioni di Cicerone, come la pro Sestio. ${ }^{14}$

Con questa connotazione negativa della vis, intesa dunque come forza antigiuridica, come violenza da contrastare attraverso uno strumento di tutela possessoria, siamo ancora nell'ambito del diritto privato, dove pure ci riconducono la fattispecie del metus nel settore negoziale e i rimedi introdotti per la tutela dell'autore del negozio nella conclusione del quale la volontà non è stata liberamente manifestata. Parlando di metus, infatti, si intende lo stato di oppressione psichica determinato da minacce tali da indurre un soggetto a concludere un negozio, provocando dunque una manifestazione di volontà negoziale viziata.

Ricostruire la storia delleditto "quod metus causa" non è facile: Ulpiano afferma che in origine (e, dobbiamo pensare, fino al I sec. d. C.) per accordare tutela a colui che veniva sottoposto a pressione per la conclusione di un negozio si contemplavano la vis e il metus, il secondo inteso quale prodotto della prima, come mostra D. 4.2.1 (Ulp. 11 aded.):

Ait praetor: quod metus causa gestum erit ratum non habebo. Olim ita edicebatur: quod vi metusve causa: vis enim fiebat mentio propter necessitatem impositam contrariam voluntati: metus instantis vel futuri periculi causa mentis trepidatio. Sed postea detracta est vis mentio ideo, quia quodcumque vi atroci fit, id metu quoque fieri videtur. ${ }^{15}$

Leditto fu introdotto a Roma nei primi decenni del I secolo a.C., elaborato e raffinato dalla giurisprudenza tardorepubblicana; in età classica furono fissati i rimedi processuali, modificati poi in epoca giustinianea. ${ }^{16}$ Il pretore intese prendere in considerazione le conseguenze della vis, introducendo una forma di tutela della vittima della violenza morale. ${ }^{17}$

13 D. 43.17. 1 pr.; cfr. Labruna (1971, pp. 87-135) per le altre fonti sul testo dell'interdetto; v. pure Labruna (1986, pp. 47-70), dove sono riprodotte alcune delle numerose recensioni al volume del 1971. Questa tesi non è accettata pacificamente dalla dottrina: vi sono, infatti, studiosi che negano una stratificazione nel testo interdittale, da ultimo Nicosia (2015); ma anche chi ritiene che l'interdetto sia assai più antico, come Falcone (1996, pp. 157-228 e 242-257). Presso gli autori citati ulteriori fonti e indicazioni bibliografiche.

14 Pro Sest. 42.91, ma anche Cic. in Verr. II.3.65.152, e, emblematico, Cic. de leg. 3.18.42: Deinceps sunt cum populo actiones, in quibus primum et maximum, vis abesto. Nihil est enim exitiosius civitatibus, nihil tam contrarium iuri ac legibus, nihil minus civile et inhumanius, quam composita et constituta re publica quicquam agi per vim.

15 "Dice il pretore: 'quanto sarà fatto a causa di timore non permetterò che consegua effetti'. Un tempo il testo edittale era così redatto: 'quanto a causa di violenza o di timore': infatti veniva fatta menzione della violenza, in ragione dell'imposizione della necessità < di agire > in modo contrario alla volontà, mentre <veniva fatta menzione> del timore <per> l'agitazione interiore causata da un pericolo attuale o futuro. In seguito, però, la menzione della violenza fu tolta, poiché quanto fatto a causa di grave violenza, è sembrato fatto anche per timore". Trad it. Schipani (2005).

16 Si v. Calore (2011) a cui rinvio per i riferimenti bibliografici anteriori.

17 Così da ultimo Calore (2011, pp. 405-421). Aderisce a questa interpretazione Cursi (2015, p. 395). La vis, la violenza fisica, con la quale si costringeva taluno a compiere un negozio, fu repressa dal diritto criminale, costituendo, appunto, una delle fattispecie del crimen vis. 
Anche la creazione di altri provvedimenti d'urgenza, altri interdetti, come quello de vi armata, che contemplava una ipotesi aggravata di violenza recata con bande armate allo scopo di spossessare taluno di un suo fondo, pure questa attestata da Cicerone, appartiene allo stesso periodo.

Dal punto di vista del diritto pubblico, le leggi che configurano la vis come integrativa di una fattispecie criminosa sono tutte dell'ultima età repubblicana, emanate, dunque, nel periodo convulso delle guerre civili: le principali, la legge Lutatia de vi (di M. Lutazio Catulo, del 78 o 77), istitutiva del tribunale straordinario per i colpevoli della rivolta di Lepido (i cittadini che con uso delle armi avessero assediato il senato, fatto violenza ai magistrati, si fossero sollevati contro la repubblica); ${ }^{19}$ la lex Plautia de vi (probabilmente del 70) per reprimere varie ipotesi di violenza politica, e forse anche di violenza contro privati.

Augusto disciplinò in modo esaustivo il crimen vis ${ }^{21}$ e introdusse la distinzione tra vis publica et privata (probabilmente con una sola lex), anche se, malgrado le copiose fonti, non è sempre facile distinguere le varie fattispecie che integravano le diverse ipotesi delittuose, per il fatto che nei Digesta (D.48.6, ad legem Iuliam de vi publica; 7 ad legem Iuliam de vi privata) a quelle originarie si sono sovrapposte quelle più ampie individuate nel corso dell'età imperiale; ${ }^{23}$ ad ogni modo non si è lontani dal vero ritenendo che fosse configurata come vis publica lattività del magistrato titolare di imperium che abusando dei propri poteri di coercizione uccidesse o facesse fustigare un cittadino romano senza tener conto della provocatio ad populum.

Le altre previsioni, ricostruibili con margini congetturali, collegano alla vis publica il turbamento del regolare svolgimento dei giudizi o delle assemblee, portare armi in pubblico, compiere saccheggi in caso di calamità, ecc., alla vis privata l’organizzazione di bande per attentare a persone $\mathrm{o}$ a proprietà altrui, il turbamento di cerimonie funebri, il sequestro di persona.

Un cenno va fatto anche alla violenza sessuale che, in base alle fonti contenute nei Digesti giustinianei, appare repressa dalle leggi de vi certamente nel III secolo come già dall’epoca tardorepubblicana, mentre forse al tempo di Augusto questo crimen fu sanzionato nell'ambito della legge de adulteriis.

18 Pro. Tull. 3.6 e passim.

19 Cic. pro Cael. 1.1.

20 Era prevista per i colpevoli la poena capitis o l'aqua et igni interdictio. Oltre al basilare Labruna (1971, pp. 50-53), v. anche Solidoro (1993, p.11); (2001, pp. 33-77); per una diversa ricostruzione delle leges de vi, v., tra gli altri, Saiz López (1992). Recentissima l'antologia di Y. Rivière (2021): alle pp. 261-288 considerazioni sul crimen vis in relazione al processo a Milone per l'uccisione di Clodio.

21 Si ricorda che il genitivo vis è molto raro: questo è, appunto, uno dei pochissimi suoi impieghi, che per lo più appartengono al campo giuridico: cfr. Forcellini, s.v. vis.

22 L'unicità della legge è ribadita di recente da Cossa (2008, pp. 209-308); ivi anche vasta e dettagliata discussione della dottrina.

23 Anche su questo aspetto v. Cossa (2008, pp. 245-252).

24 D.48.6.7 (Ulp. 8 de off. proc.): Lege lulia de vi publica tenetur, qui, cum imperium potestatemve haberet, civem Romanum adversus provocationem necaverit verberaverit iusseritve quid fieri aut quid in collum iniecerit, ut torqueatur ("Secondo la legge Giulia è tenuto per violenza pubblica colui che, dotato di imperium e potestà, contravvenendo alla provocatio avrà ucciso, fustigato un cittadino romano, o avrà ordinato che gli sia fatto qualcosa o che gli sia messo qualcosa al collo perché venga torturato").

25 Così Santalucia (1998, pp. 198-202); Santalucia (2013, pp. 83-85). La pena prevista era l'aqua et igni interdictio in caso di violenza pubblica, per la vis privata la confisca della terza parte del patrimonio e l'interdizione dalle cariche pubbliche.

26 Cfr. D. 48.5 .30 (29).9 (Ulp. 4 de adult.): Eum autem, qui per vim stuprum intulit vel mari vel feminae, sine praefinitione huius temporis accusari posse dubium non est, cum eum publicam vim committere nulla dubitatio est (“Anche colui che con la violenza ha commesso stupro contro un maschio o una donna non v'è dubbio che possa venire accusato senza la prescrizione di questo tempo, mentre non si dubita che egli commetta una violenza pubblica"); e D. 48.6.3.4 (Marcian. 14 inst.): Praeterea punitur huius legis poena, qui puerum vel feminam vel quemquam per vim stupraverit (“Inoltre è punito con la pena di questa legge chi avrà stuprato con la violenza un fanciullo, una 
Come detto è difficile dai passi dei Digesti ricostruire le nuove previsioni di ampliamento della vis publica e privata di Augusto: una costituzione di Marco Aurelio, però ci è nota dalle Pandette e conviene qui ricordarla, D.48.7.7 (Callistratus 5 de cognitionibus):

Creditores si adversus debitores suos agant, per iudicem id, quod deberi sibi putant, reposcere debent: alioquin si in rem debitoris sui intraverint id nullo concedente, divus Marcus decrevit ius crediti eos non habere. Verba decreti haec sunt. "Optimum est, ut, si quas putas te habere petitiones, actionibus experiaris: interim ille in possessione debet morari, tu petitor es". Et cum Marcianus diceret: "Vim nullam feci": Caesar dixit: "Tu vim putas esse solum, si homines vulnerentur? Vis est et tunc, quotiens quis id, quod deberi sibi putat, non per iudicem reposcit...". ${ }^{27}$

Il principe nega il diritto di credito a un tale che si era rifatto direttamente sui beni del debitore senza adire all'autorità statale. Di fronte all'affermazione di Marciano (il creditore): "Non ho commesso alcuna violenza", Marco Aurelio risponde: "Credi tu che vi sia violenza solo se degli uomini sono colpiti? Violenza si ha ogni volta che qualcuno non richiede attraverso un giudice quello che gli è dovuto", con ciò stabilendo gli inizi della fine dell'autotutela, e mostrando come la nozione di vis si sia fortemente ampliata dall'età repubblicana.

Sappiamo che Costantino abolì la differenza tra vis publica e vis privata inasprendo la pena, ora quella capitale; in età giustinianea si riprende la distinzione ma unitamente al mutamento di prospettiva non è possibile distinguere con chiarezza le fattispecie: sappiamo che la vis publica fu equiparata alla vis armata, quella privata alla vis sine armis.

In conclusione, va ricordata solo un'altra fonte che illumina sulla configurazione del crimen vis nellepoca postgiustinianea. Isidoro di Siviglia tra VI e VII secolo scrive nelle sue Etymologiae (5.26.4): Vis est virtus potestatis, per quam causa sive res vel aufertur vel extorquetur. "Si definisce vis, ossia violenza, il potere dell'autorità di ottenere o estorcere una causa o una cosa". Quindi si configura come vis l'abuso di potere da parte di una pubblica autorità. Il diritto criminale rispecchiato nell'opera di Isidoro è destinato sia ai Goti sia ai Romani, e benché in questa parte della sua opera, relativa al crimen vis (il paragrafo 4 con Etym. 5.26.5 e 6), il vescovo si basi sulle Pauli Sententiae, altra importante fonte postclassica sulla lex de vi, nel richiamo alla classificazione della vis come publica e privata, ${ }^{29}$ proprio il distacco che si rileva nel passo 5.26 .4 dal dettato delle sentenze pseudopaoline permette di mettere in luce un altro aspetto del lavoro intellettuale del sivigliano, che avrebbe forse addirittura elaborato consapevolmente

donna o chiunque altro"); si v. Botta (2004; 2011, pp. 85-147); Rizzelli (2012, pp. 295-377).

27 "Se i creditori agiscono contro i loro debitori, devono richiedere attraverso un giudice quello che ritengono sia dovuto loro: diversamente se si sono immessi nel patrimonio del debitore senza che nessuno (a seguito di processo) lo abbia autorizzato, il divino Marco (Aurelio) decretò che questi non avessero (più) il diritto di credito. Ecco le parole del decreto: 'Ottima cosa è esperire delle azioni se ritieni di avere delle pretese; ma finché tu sei nella veste di richiedente, l'altro deve rimanere nel suo possesso'. E poiché Marciano disse 'Non ho compiuto nessuna violenza', Cesare affermò: 'Tu ritieni che sia violenza soltanto se degli uomini vengono colpiti? Violenza è anche ogni volta che qualcuno non richiede attraverso il giudice ciò che ritiene gli sia dovuto ...”.

28 Così Martini e Pietrini (2011, pp. 57-80, spec. pp. 77-80).

29 Pauli Sent. 5.26.1: Lege Iulia de vi publica damnatur, qui aliqua potestate praeditus civem Romanum antea ad populum, nunc imperatorem appellantem necaverit necarive iusserit, torserit, verberaverit, condemnaverit inve publica vincula duci iusserit ... (“È condannato secondo la legge Giulia sulla violenza pubblica chiunque, investito di autorità, avrà ucciso, ordinato di uccidere, torturato, fustigato, condannato, ordinato di gettare in carcere un cittadino romano che una volta si appellava al popolo, oggi che si sia appellato al princeps"); Pauli Sent. 5.26.3: Lege lulia de vi privata tenetur, qui quem armatis hominibus possessione, domo villa agrove deiecerit, expugnaverit obsederit, clauserit, ... ("È tenuto secondo la legge Giulia sulla violenza privata chiunque con uomini armati avrà scacciato qualcuno da un suo possesso, casa, villa o campo, avrà preso questi luoghi, o li avrà assediati..."); cfr. Isid, Etym. 5.26.5-6: 5. Vis privata est, si quisque ante iudicium armatis hominibus quemquam a suo deiecerit vel expugnaverit. 6. Vis publica est, si quis civem ante populum vel iudicem vel regem appellantem necaverit, aut torserit sive verberaverit vel vinxerit. 
e in maniera autonoma alcuni concetti che trovava nelle fonti giuridiche. ${ }^{30}$ Questa tarda riflessione sul concetto di vis mostra che ancora nel VII secolo si continuava a ragionare su temi, concetti e termini centrali per la convivenza civile tentando di apportare nuove idee al diritto romano codificato.

\section{Bibliografia citata}

》 Amodio, M. (2017). Violenza ed emozioni. II linguaggio delle immagini nella Roma tardoantica. In S. Botta, M. Ferrara e A. Saggioro (Eds.), La storia delle religioni e la sfida dei pluralismi. Atti del Convegno della Società Italiana di Storia delle Religioni-Roma, Sapienza, 8-9 aprile 2016 (342-350). Brescia: Morcelliana.

» Balzarini, M. (1993). S.v. Violenza (dir. rom.). In Enciclopedia del diritto (46, 830-843). Milano: Giuffrè.

" Botta, F. (2004). "Per vim inferre". Studi su "stuprum" violento e "raptus" nel diritto romano e bizantino. Cagliari: AV.

" Botta, F. (2011). "Stuprum per vim illatum". Violenza e crimini sessuali nel diritto classico e dell'occidente tardoantico. In F. Botta, F. Lucrezi e G. Rizzelli, G. (Eds.), Violenza sessuale e società antiche. Profili storico-giuridici (85-147). Lecce: Grifo.

"Briguglio, F. (2010). Servio Sulpicio e la definizione di tutela: "vis ac potestas" o "ius ac potestas"?. In C. Russo Ruggeri (Ed.), Scritti in onore di A. Metro I (163-179). Milano: Giuffrè.

"Calore, E. (2011). Actio quod metus causa. Tutela della vittima e azione in rem scripta. Milano: Giuffrè.

»Cantarella, E. (2011). I supplizi capitali. Origine e funzioni delle pene di morte in Grecia e a Roma. Milano: Feltrinelli.

"Cossa, G. (2008). Attorno ad alcuni aspetti della "lex Iulia de vi publica et privata". Studia et Docum. Hist. et luris, 74, 209-308.

"Cursi, M. F. (2015): “Actio quod metus causa” e le azioni “miste”, rec. a E. Calore (2011). Index, 43, 393-401.

" Falcone, G. (1996). Ricerche sull'origine dell'interdetto “uti possidetis". Annali dell'Università di Palermo, 44, 5-360.

" Labruna, L. (1971). “Vim fieri veto”. Alle radici di una ideologia. Napoli: Jovene.

"Labruna, L. (1986). Tutela del possesso fondiario e ideologia repressiva della violenza nella Roma repubblicana. Napoli: Jovene.

»Magugliani, L. (Trad.). (1979). Esiodo, Le opere e i giorni. Milano: Rizzoli.

» Martini, R., Pietrini, S. (2011). Cognizioni giuridiche nel libro V delle "Etymologiae" di Isidoro di Siviglia. In G. Bassanelli Sommariva e S. Tarozzi (Eds.). Ravenna Capitale. Uno sguardo ad Occidente. Romani e Goti - Isidoro di Siviglia (57-80). Sant'Arcangelo di Romagna: Maggioli.

" McClintock, A. (2010). Servi della pena. Condannati a morte nella Roma imperiale. Napoli: Edizioni Scientifiche Italiane.

»McClintock, A. (2015). Per un'iconologia dei supplizi. Rivista di Diritto Romano, 15, 1-10. 
» Mittica, M. P. (2011). Frammenti di analisi letterarie del rapporto tra giustizia e diritto. In A. Cantaro (Ed.), Giustizia e diritto nella scienza giuridica contemporanea (156-169). Torino: Giappichelli.

"Nicosia, G. (2015). “Vitiosa possessio" e “iniusta possessio”. IURA, 43, 1-27.

» Pietrini, S. (2014). “Iudex”, “accusator” e "calumniator” in Isidoro di Siviglia e nel diritto visigoto. Index, 42, 446-46o.

»Querzoli, S. (2004). 'Vis', ‘ius', 'natura'. La correttezza giuridica della legittima difesa dalla teorizzazione ciceroniana alla riflessione giurisprudenziale. Ostraka, 13, 277-284.

» Rivière, Y. (2021). Histoire du droit pénal romain. De Romulus à Justinien. Paris: Les Belles Lettres.

» Rizzelli, G. (2012). La violenza sessuale su donne nell'esperienza di Roma antica. Note per una storia degli stereotipi. In E. Höbenreich, V. Kühne e F. Lamberti (Eds.), El Cisne II. Violencia, proceso y discurso sobre género (295-377). Lecce: Grifo.

» Saiz López, V. (1992). La represión de la vis en época republicana. Anales de la Universidad de Alicante. Facultad de Derecho, 7, 191-209.

»Santalucia, B. (1998). Diritto e processo penale nell'antica Roma. Milano: Giuffrè.

»Santalucia, B. (2013). La giustizia penale in Roma antica. Bologna: Il Mulino.

»Schipani, S. (Trad.). (2005). Iustiniani Augusti Digesta Seu Pandectae, Testo e Traduzione, vol. I. Milano: Giuffrè. Anche on line all'indirizzo http://dbtvm1.ilc.cnr.it/digesto/ (consultato il 7 febbraio 2021)

»Solidoro, L. (1993). La repressione della violenza nel diritto penale romano: appunti dalle lezioni. Napoli: Jovene.

»Solidoro, L. (2001). La repressione della criminalità organizzata tra diritto romano e categorie moderne. Criteri di impostazione della ricerca. Vincula iuris. Studi in onore di Mario Talamanca, VII (33-77). Napoli: Jovene.

»Viarengo, G. (2015). Studi sulla tutela dei minori. Torino: Giappichelli.

»Vismara, C. (1991). Il supplizio come spettacolo. Roma: Quasar. 